\title{
The Logarithmic Mean Revisited
}

\section{Graham Jameson and Peter R. Mercer}

Abstract. We present a simple method for establishing several inequalities relating to logarithmic means.

The logarithmic mean of two distinct positive numbers $a, b$ is

$$
L(a, b)=\frac{b-a}{\log b-\log a} .
$$

The basic inequality for the logarithmic mean states that it sits between the geometric mean $G(a, b)=(a b)^{1 / 2}$ and the arithmetic mean $A(a, b)=\frac{1}{2}(a+b)$ :

$$
G(a, b) \leq L(a, b) \leq A(a, b) .
$$

We note here that the special case $a=1$ (with $b$ replaced by $x$ ) says, for all $x>0$,

$$
x^{1 / 2} \leq \frac{x-1}{\log x} \leq \frac{1}{2}(x+1) .
$$

Conversely, the substitution $x=b / a$ transforms (2) into (1), so in fact the statements are equivalent. In this way, (1) reduces to inequalities in terms of a single variable $x$.

Numerous proofs of (1) have been given. The recent note [10] presents a proof using Cauchy's mean value theorem (though in fact the ordinary mean value theorem works equally well). Other proofs can be seen, for example, in [4], [5], and [11, pp. 272-273].

The note [10] goes on to give proofs, again using Cauchy's mean value theorem, of some more intricate inequalities, which had been obtained by quite complicated methods in earlier papers ([6], [8], [9]). We state them here, writing just $L$ for $L(a, b)$, and similarly $A$ and $G$.

$$
\begin{gathered}
L \leq \frac{2}{3} G+\frac{1}{3} A . \\
L \geq G^{2 / 3} A^{1 / 3} . \\
L \leq M_{1 / 3} .
\end{gathered}
$$

Here $M_{1 / 3}$ is the case $p=1 / 3$ of the power mean $M_{p}(a, b)=\left[\frac{1}{2}\left(a^{p}+b^{p}\right)\right]^{1 / p}$.

It was pointed out in [7] that inequalities (3) and (4) together imply that the number $1 / 3$ is optimal in each case.

Here we give an exposition of a pleasantly simple method for these inequalities, along with a few related ones. The strategy is to translate the required inequality into an equivalent statement in terms of sinh and cosh, which, with luck, is easily proved using power series. The use of this technique for the purpose of obtaining inequalities for means is well established: for example, see [1]. In relation to the inequalities considered here, a proof of (1) by this method was outlined briefly in [3], and proofs of (3), (4), and (5) were indicated rather sketchily in [12]. We present full proofs, starting with (1). 
Proof of (1). We have seen that it is sufficient to prove (2). In (2), substitute $x=e^{2 y}$ : the statement becomes (for all $y$ )

$$
e^{y} \leq \frac{e^{2 y}-1}{2 y} \leq \frac{1}{2}\left(e^{2 y}+1\right) .
$$

After division by $e^{y}$, this says

$$
1 \leq \frac{\sinh y}{y} \leq \cosh y .
$$

So (1) is equivalent to the beautifully simple statement (6), which can be read off with no further work from the series expansions

$$
\begin{aligned}
& \frac{\sinh y}{y}=1+\frac{y^{2}}{3 !}+\frac{y^{4}}{5 !}+\cdots, \\
& \cosh y=1+\frac{y^{2}}{2 !}+\frac{y^{4}}{4 !}+\cdots .
\end{aligned}
$$

We now show how this method delivers (3), (4), and (5).

Proof of (3). We do not need to know the factor $1 / 3$ in advance: we can let it emerge from the reasoning. So consider (3) in the form $L \leq(1-p) G+p A$, where $p$ is to be found. For the strongest possible result, we must choose $p$ as small as possible. As before, it is sufficient to prove the case $a=1$, in other words (with $b$ replaced by $x$ )

$$
\frac{x-1}{\log x} \leq(1-p) x^{1 / 2}+\frac{p}{2}(x+1) .
$$

The substitution $x=e^{2 y}$ tranforms this into

$$
\frac{e^{2 y}-1}{2 y} \leq(1-p) e^{y}+\frac{p}{2}\left(e^{2 y}+1\right),
$$

equivalently,

$$
\frac{\sinh y}{y} \leq(1-p)+p \cosh y .
$$

The article [12] does not include a proof of (9), but this is again achieved very easily by comparing power series (it is difficult to imagine a simpler proof). Indeed,

$$
(1-p)+p \cosh y=1+p\left(\frac{y^{2}}{2 !}+\frac{y^{4}}{4 !}+\cdots\right)
$$

Statement (9) will be assured if the coefficients are no smaller than the corresponding ones in (7). The $y^{2}$ term requires that $p / 2 \geq 1 / 6$, so $p \geq 1 / 3$. The $y^{2 n}$ term requires $p /[(2 n) !)] \geq 1 /[(2 n+1) !]$, or $p \geq 1 /(2 n+1)$. So (9) holds with $p=1 / 3$.

At the same time, it is clear that (9) will fail for some $y$ if $p<1 / 3$, since $(1-p)+$ $p \cosh y=1+\frac{p}{2} y^{2}+O\left(y^{4}\right)$, while $(\sinh y / y)=1+\frac{1}{6} y^{2}+O\left(y^{4}\right)$. 
Proof of (4). For this, given the previous proof and the remark in [7], we will accept the index $1 / 3$ from the start. As before, (4) is equivalent to the case $a=1$, that is,

$$
\frac{x-1}{\log x} \geq x^{1 / 3}\left[\frac{1}{2}(x+1)\right]^{1 / 3} .
$$

The substitution $x=e^{2 y}$ transforms this into

$$
\frac{e^{2 y}-1}{2 y} \geq e^{2 y / 3}\left(\frac{1}{2}\left(e^{2 y}+1\right)\right)^{1 / 3} .
$$

After division by $e^{y}$, this is equivalent to

$$
\frac{\sinh y}{y} \geq(\cosh y)^{1 / 3},
$$

hence to

$$
\left(\frac{\sinh y}{y}\right)^{3} \geq \cosh y
$$

This time, comparison of the coefficients will cost us a little more work. For readers with the appetite for it, here are the details. Note that

$$
(\sinh y)^{3}=\frac{1}{8}\left(e^{y}-e^{-y}\right)^{3}=\frac{1}{4} \sinh 3 y-\frac{3}{4} \sinh y=\sum_{n=0}^{\infty} c_{2 n} y^{2 n+3},
$$

where

$$
c_{2 n}=\frac{3^{2 n+3}-3}{4(2 n+3) !} .
$$

We need to know that $c_{2 n} \geq 1 /[(2 n) !]$ for each $n \geq 0$. This equates to saying that $u_{n} \geq v_{n}$, where

$$
u_{n}=3^{2 n+3}-3, \quad v_{n}=4(2 n+1)(2 n+2)(2 n+3) .
$$

To start, we have $u_{0}=v_{0}=24$ and $u_{1}=v_{1}=240$. For all $n \geq 1$, it is clear that $u_{n+1} / u_{n}>9$, while

$$
\frac{v_{n+1}}{v_{n}}=\frac{(2 n+4)(2 n+5)}{(2 n+1)(2 n+2)} \leq \frac{6 \times 7}{3 \times 4}=\frac{7}{2},
$$

so indeed $u_{n}>v_{n}$, as required.

At the same time, if $q<3$, then by (7) and the binomial series, we have $(\sinh y / y)^{q}$ $=1+\frac{q}{6} y^{2}+O\left(y^{4}\right)<\cosh y$ for sufficiently small $y$, so $1 / 3$ is optimal in (4).

Remark. The inequality (10) is given in [11, p. 270], without any mention of the equivalence with (4). Mitrinovic's method is by the mean value theorem, twice differentiating the function $y-\sinh y(\cosh y)^{-1 / 3}$. Readers may wish to try this and compare with the series method. Another method based on differentiation can be seen in [2]. 
Proof of (5). After replacing $a$ and $b$ by 1 and $x$, and substituting $x=e^{2 y}$, we see that (5) is equivalent to

$$
\frac{\sinh y}{y} \leq\left(\cosh \frac{1}{3} y\right)^{3}=\frac{1}{4} \cosh y+\frac{3}{4} \cosh \frac{1}{3} y .
$$

The series expression for the right-hand side is $\sum_{n=0}^{\infty} d_{2 n} y^{2 n}$, where

$$
d_{2 n}=\frac{1}{(2 n) !}\left(\frac{1}{4}+\frac{3}{4.3^{2 n}}\right) \text {. }
$$

Comparing with (7), we need to know that $d_{2 n} \geq 1 /[(2 n+1) !]$ for each $n$. This equates to

$$
\frac{1}{4}+\frac{3}{4.3^{2 n}} \geq \frac{1}{2 n+1}
$$

This is much easier than the previous proof! When $n=0$, both sides are 1 , and when $n=1$, both sides are $1 / 3$. The required inequality is obvious for $n \geq 2$.

It is well known that $M_{p}$ increases with $p$. By the binomial series again, $(\cosh p y)^{1 / p}$ $=1+\frac{p}{2} y^{2}+O\left(y^{4}\right)$, so we cannot replace $1 / 3$ by $p<1 / 3$ in (5).

We describe some further inequalities for means of assorted types delivered with minimal effort by this method. First, the following comparison between the upper bounds in (3) and (5):

$$
M_{1 / 3} \leq \frac{2}{3} G+\frac{1}{3} A .
$$

This statement does not involve $L$, but of course, together with (5), it implies (3).

Proof of (12). Again replacing $a$ and $b$ by 1 and $x$, and substituting $x=e^{2 y}$, we see that (12) is equivalent to

$$
\left(\cosh \frac{1}{3} y\right)^{3} \leq \frac{2}{3}+\frac{1}{3} \cosh y .
$$

Now $\left(\cosh \frac{1}{3} y\right)^{3}=\frac{1}{4} \cosh y+\frac{3}{4} \cosh \frac{1}{3} y$, so the statement is equivalent to

$$
9 \cosh \frac{1}{3} y \leq 8+\cosh y \text {. }
$$

The constant term is 9 on both sides, and for $n \geq 1$, the coefficient of $y^{2 n}$ on the left-hand side is

$$
\frac{1}{3^{2 n-2}(2 n) !}
$$

which is not greater than $1 /[(2 n) !]$.

Remark. This raises the obvious question of how $M_{p}$ compares with $(1-p) G+p A$ for general $p$. Now $M_{1}=A$ and, on writing it out, one sees that $M_{1 / 2}=\frac{1}{2} G+\frac{1}{2} A$. A proof like the one just given (admittedly with a little more work) shows that $M_{2 / 3} \geq$ $\frac{1}{3} G+\frac{2}{3} A$. It seems natural to conjecture that $M_{p} \leq(1-p) G+p A$ for $0<p \leq 1 / 2$ and that the opposite holds for $1 / 2<p \leq 1$. However, a word of caution is in order. All these means, unlike $L$, make sense for three or more numbers. Simple examples show that for three numbers, $M_{1 / 2}$ can be either greater or less than $\frac{1}{2} G+\frac{1}{2} A$, and equally, $M_{1 / 3}$ can be greater or less than $\frac{2}{3} G+\frac{1}{3} A$. 
For the next result, let us work from the other end. Start from the obvious inequality $\cosh y \geq 1+\frac{1}{2} y^{2}$. How does this translate into a statement about means when the steps above are applied in the reverse order? Multiplication by $e^{y}$ transforms it into

$$
\frac{1}{2}\left(e^{2 y}+1\right) \geq\left(1+\frac{1}{2} y^{2}\right) e^{y} .
$$

With the substitution $x=e^{2 y}$, this becomes

$$
\frac{1}{2}(x+1) \geq\left(1+\frac{1}{8}(\log x)^{2}\right) x^{1 / 2},
$$

so, finally substituting $x=b / a$, we conclude

$$
A \geq\left(1+\frac{1}{8}(\log b-\log a)^{2}\right) G .
$$

This is an enhanced version of the basic inequality $A \geq G$. It was proved, by a more elaborate method, in [13]. Clearly, it could be enhanced further, at the cost of greater complication, by incorporating further terms of the cosh series.

Readers will be able to verify that, in the same way, the inequality $\frac{1}{y} \sinh y \geq$ $1+\frac{1}{6} y^{2}$ translates into

$$
L \geq\left(1+\frac{1}{24}(\log b-\log a)^{2}\right) G
$$

bringing us neatly back to the logarithmic mean.

Added in proof: The conjecture relating to $M_{p}$ has been proved by Gord Sinnamon.

\section{REFERENCES}

1. Alzer, H. (1986). Ungleichungen für Mittelwerte. Arch. Math. 47(5): 422-426.

2. Anderson, G., Vamanamurthy, M., Vuorinen, M. (2006). Monotonicity rules in calculus. Amer. Math. Monthly. 113(9): 805-816.

3. Bhatia, R. (2008). The logarithmic mean. Resonance. 13(6): 583-594.

4. Bruce, I. (1997). The logarithmic mean. Math. Gaz. 81(490): 89-92.

5. Burk, F. (1987). The geometric, logarithmic, and arithmetic mean inequality, Amer. Math. Monthly. 94(6): 527-528.

6. Carlson, B. C. (1972). The logarithmic mean. Amer. Math. Monthly. 79(6): 615-618.

7. Jameson, G. (2017). Feedback on 101.07. Math. Gaz. 101(552): 545.

8. Leach, E. B., Sholander, M. C. (1983). Extended mean values II. J. Math. Anal. Appl. 92(1): 207-223.

9. Lin, T.-P. (1974). The power mean and the logarithmic mean. Amer. Math. Monthly. 81(8): 879-883.

10. Mercer, P. R. (2017). Cauchy's mean value theorem meets the logarithmic mean. Math. Gaz. 101(550): $108-115$.

11. Mitrinovic, D. S. (1970). Analytic Inequalities. Berlin, Heidelberg, New York: Springer.

12. Zhu, L. (2005). From chains for mean value inequalities to Mitrinovic's problem II. Int. J. Math. Education in Science and Technology. 36(1): 118-125.

13. Zou, L., Jiang, Y. (2015). Improved arithmetic-geometric mean inequality and its application. J. Math. Ineq. 9(1): 107-111.

Department of Mathematics and Statistics, Lancaster University, Lancaster LA1 4YF, United Kingdom g.jameson@lancaster.ac.uk

Department of Mathematics, Buffalo State College, Buffalo NY 14221, USA mercerpr@buffalostate.edu 